\title{
Discussion of Bickel and Rosenblatt's work on Global Measures of Deviations for Density Estimates
}

\section{By Peter Bickel}

The paper [1] of Murray Rosenblatt's, which I coauthored, seems to have had a reasonably long shelf life. I believe Murray enjoyed working on this paper-I certainly did! The question posed by Murray was that of obtaining asymptotic approximations to the distribution of the maximum deviation of a kernel density estimate $\hat{f}_{n}$ of an unknown density $f$ based on a sample $X_{1}, \ldots, X_{n}$. If a limit distribution existed for

$$
\sigma_{n}\left[\max _{0 \leq x \leq 1}\left\{\frac{\left|\hat{f}_{n}(x)-f(x)\right|}{\sqrt{\hat{f}_{n}(x)}}\right\}-\mu_{n}\right],
$$

where $\mu_{n}, \sigma_{n}>0$ are normalizing constants not depending on $f$, then it is clear how to

(a) construct approximate uniform confidence bands for $f$ of the form $\hat{f}_{n} \pm c_{n} \sqrt{\hat{f}_{n}}$.

(b) construct goodness of fit tests of point hypotheses $H: f=f_{0}$ using

$$
\max _{x} \frac{\left|\hat{f}_{n}(x)-f_{0}(x)\right|}{\sqrt{f_{0}(x)}}
$$

as a test statistic.

The heuristic that Murray advanced was the following. If $U_{n}(x)$ denotes the stochastic process defined by

$$
U_{n}(x) \equiv \sqrt{n h_{n}} \frac{\left(\hat{f}_{n}(x)-f(x)\right.}{\sqrt{\hat{f}(x)}},
$$

then $U_{n}\left(x_{1}\right), \ldots, U_{n}\left(x_{k}\right)$ are asymptotically iid Gaussian and $U_{n}(x)$ behaves locally like a stationary Gaussian process. Hence one would expect that the statistic based on $\max _{0 \leq x \leq 1} U_{n}(x)$ would behave like the corresponding statistic for a stationary Gaussian process over an increasing stretch of the real line; namely the limit would have a Gumbel distribution. Murray and I were able to show this using strong embedding of the empirical process techniques.

We also studied another potential test statistic, the $L_{2}$ deviation, defined by

$$
\int_{0}^{1} \frac{\left(\hat{f}(x)-f_{0}(x)\right)^{2}}{f_{0}(x)} d x
$$

We showed that under strong conditions, this statistic had a limiting Gaussian distribution consistent with its $\chi^{2}$ like nature. Following this up in [5], Rosenblatt showed that this result held under very weak conditions in view of the representation of the statistic as a $U$ statistic with a kernel dependent on the sample size. This was indeed a nice and useful observation.

Subsequently, we used our methods to consider the testing problem and examined the rate at which alternatives tend to the hypothesis with non-negligible power. Not surprisingly, the rate is less than $n^{-\frac{1}{2}}$ which is what one obtains for Kolmogorov-Smirnov and similar tests, although the quadratic deviation is consistently better. However, as results of Ingster and Suslina [4] show, minimax results for different smoothness classes of alternatives are obtained for tests which resemble quadratic density deviation measures. These results clearly depend on what class of alternatives is 
considered, $\left\{f:\left\|F-F_{0}\right\|_{\infty} \geq \delta\right\}$ where $F$ is the cdf and $f$ is the density is suitable for KS tests while $\left\{f:|f(x)-f(y)| \leq M|x-y|^{\alpha}\right.$ all $\left.x, y,\left\|f-f_{0}\right\|_{2} \geq \delta\right\}$ is suitable for the test of [5].

Extensions of this work to the dependent and multivariate cases, and processes other than kernel density ones were developed quickly by Rosenblatt and others [6]. Murray and I only had one further paper in this area, [2], although we had certainly corresponded on [5] and others. The second paper was more probabilistic and derived results on the maxima of Gaussian random fields, which were later obtained more generally by Hogan and Siegmund [4] using a different method. We envisaged these results to be applied to the extension of the results of [1] to the multivariate case.

It is clear from all of the follow-up work that Rosenblatt's initial question was important and his intuition perfect.

\section{References}

[1] Bickel, P.J. and Rosenblatt M. (1973). On some global measures of the deviations of density function estimates, Ann. Statist. 1, 1071-1095.

[Correction: Ann. Statist. 3 (1975), no. 6, 1370].

[2] Bickel P.J. and Rosenblatt M. (1973). Two-dimensional random fields, Multivariate Analysis III, 3-15, Academic Press NY.

[3] Hogan M.L. and Siegmund D. (1986). Large deviations for the maxima of some random fields, Advances in Applied Probability 7, 2-22.

[4] Ingster Y. I. and Suslina, I. A. (2000). Minimax nonparametric hypothesis testing for ellipsoids and Besov bodies. ESAIM Probab. Statist. 4, 53-135.

[5] Rosenblatt M. (1975). A quadratic measure of deviation of two-dimensional density estimates and a test of independence, Ann. Statist. 3, 1-14.

[6] Rosenblatt, M. (1976). On the maximal deviation of $k$-dimensional density estimates, Ann. Probab. 4, 1009-1015. 